\title{
PRMT1 regulates tumor growth and metastasis of human melanoma via targeting ALCAM
}

\author{
LEI LI ${ }^{1,2}$, ZHENGWEN ZHANG ${ }^{2}$, TENGXIAO MA ${ }^{2}$ and RAN HUO ${ }^{1}$ \\ ${ }^{1}$ Department of Aesthetic Plastic and Burn Surgery, Shandong Provincial Hospital, Shandong University, Jinan, Shandong \\ 250000; ${ }^{2}$ Department of Plastic and Reconstructive Surgery, Henan Provincial People's Hospital, \\ Zhengzhou, Henan 450000, P.R. China
}

Received June 25, 2015; Accepted April 28, 2016

DOI: $10.3892 / \mathrm{mmr} .2016 .5273$

\begin{abstract}
Overexpression of protein arginine methyltransferases (PRMTs) is associated with various types of cancer. The present study aimed to determine the expression level of PRMT1 in human melanoma and investigate its biological function. The clinical significance of PRMT1 was determined by screening the Oncomine database, and the increased expression of PRMT in melanoma was confirmed by western blot analysis. Furthermore, the current study demonstrated that PRMT1 was overexpressed in melanoma cell lines compared with human immortalized keratinocytes and PIG1 immortalized human melanocytes. Silencing PRMT1 in A375 and Hs294T cells significantly suppressed tumor growth and metastatic ability of the melanoma cell line compared with the negative control. These changes were in accordance with the upregulation of the cadherin 1 level and downregulation of several metastatic-associated genes determined by a quantitative polymerase chain reaction array. Liquid chromatography-mass spectrometry demonstrated that activated leukocyte cell adhesion molecule (ALCAM) may be a direct target of PRMT1, and the interaction was confirmed by co-immunoprecipitation. Compared with negative controls, the protein level of ALCAM was decreased following the silencing of PRMT1, and re-expression of ALCAM in A375/shPRMT1 or Hs294T/shPRMT1 cells using an expression vector restored the colony formation and metastatic ability of the cells. In conclusion, the current results indicated that PRMT1 is overexpressed in human melanoma, and may regulate tumor growth and metastasis via targeting ALCAM.
\end{abstract}

Correspondence to: Dr Ran Huo, Department of Aesthetic Plastic and Burn Surgery, Shandong Provincial Hospital, Shandong University, 324 Jing Five Wei Seven Road, Jinan, Shandong 250000, P.R. China

E-mail: huoran@medmail.com.cn

Key words: protein arginine methyltransferase (PRMT1), melanoma, tumor growth, metastasis, activated leukocyte cell adhesion molecule (ALCAM)

\section{Introduction}

Melanoma is the most serious type of skin cancer, with an increasing incidence worldwide (1). The transition from the radial growth phase to the vertical growth phase is responsible for the majority of cancer-associated mortalities caused by melanoma (2). Sun exposure and acquired genetic alterations are two well-established etiologies for melanoma, and previous studies have revealed that congenital germline mutation of certain critical genes, such as cyclin-dependent kinase inhibitor 2A, is another risk factor (3-5). The diagnostic technology and health consciousness regarding melanoma have greatly improved in recent years and, furthermore, the surgical techniques and adjuvant treatment measures have progressed $(6,7)$. However, the survival rate of patients with melanoma remains a cause for concern, and the majority of patients will exhibit recurrence within 5 years (8). Thus, effective therapeutic measures based on novel molecular targets that are involved in the progress of melanoma require investigation.

Histone arginine methylation is a dynamic process that transfers a methyl group from $\mathrm{S}$-adenosylmethionine (AdoMet) to the guanidine group of targeted arginine residues (9). Protein arginine methylation regulates multiple biological processes, including gene transcription and signaling transduction (10). This modification process is mainly catalyzed by nine protein arginine methyltransferases (PRMTs) (11). Dysregulation of PRMTs has previously been identified in several human types of tumor (11). PRMT5 was previously identified as a prognostic marker of lung cancer, which regulates tumor growth and the cell cycle (12). Additionally, PRMT2 regulates the activity of E2F transcription factor and the estrogen receptor $\alpha$ status in breast cancer $(13,14)$. Regarding melanoma, overexpression of PRMT5 was correlated with tumor progression and metastasis; furthermore, PRMT5 was demonstrated to regulate the level of microphthalmia-associated transcription factor and the cell cycle regulator, cyclin-dependent kinase inhibitor 1B (p27) $(15,16)$. However, whether PRMT1 regulates the aggressive behavior of human melanoma remains elusive. The present study aimed to clarify the role of PRMT1 in melanoma and to explore the potential mechanisms involved. 


\section{Materials and methods}

Ethical statement. All specimens were obtained with the informed consents of patients prior to surgery. All human and animal studies had been approved by the Ethical Committee of Shandong Provincial Hospital, Shandong University School of Medicine (Jinan, China), and were therefore performed in accordance with the ethical standards of the 1964 Declaration of Helsinki and its later amendments.

Clinical specimens and cell culture. Melanoma specimens and adjacent normal tissues $(n=28)$ were obtained from Shandong Provincial Hospital (Jinan, China). Human melanoma cell lines, A375, Hs294T and SK-MEL-28, and human immortalized keratinocytes (HaCaTs) and immortalized human melanocyte cell line, PIG1 (control cell lines), were obtained from the American Type Culture Collection (Manassas, VA, USA). All cells were cultured in Dulbecco's modified Eagle's medium (DMEM; Gibco; Thermo Fisher Scientific, Inc., Waltham, MA, USA) containing 10\% fetal bovine serum (FBS) at $37^{\circ} \mathrm{C}$ in a $5 \% \mathrm{CO}_{2}$ atmosphere.

Lentiviral transfection for stable expression clones. Lentivirus products expressing short hairpin RNA targeting (shPRMT1) or negative control (shNC) were purchased from GeneChem Co., Ltd. (Shanghai, China). The interference effect of three shRNAs was verified and the effective interference shRNA was used for silencing PRMT1. The sequence in the PRMT1 gene recognized by shPRMT1 is 5'-GACATGACATCCAAAGACT-3'. Stable cell lines were obtained according to the manufacturer's infection and selection protocol.

Immunoprecipitation (IP), liquid chromatography-mass spectrometry $(L C-M S)$ and western blot analysis. IP was conducted using protein G-agarose (EMD Millipore, Billerica, MA, USA). LC-MS was used to detect proteins that interact with PRMT1. For western blot analysis, cells were lysed with radioimmunoprecipitation assay buffer (Beijing Solarbio Science \& Technology Co., Ltd., Beijing, China) supplemented with a protease inhibitor cocktail (Pierce; Thermo Fisher Scientific, Inc., Waltham, MA, USA) and protein concentration was determined by Bradford assay. Protein lysates $(20 \mu \mathrm{g})$ were separated by sodium dodecyl sulfate polyacrylamide gel electrophoresis and resulting gels were transferred to a polyvinylidene difluoride membrane (EMD Millipore). Indicated primary antibodies were incubated with the membranes overnight at $4^{\circ} \mathrm{C}$ following blocking with skimmed milk powder for $2 \mathrm{~h}$ at room temperature. Protein bands were visualized using Pierce ECL Western Blotting Substrate (Pierce; Thermo Fisher Scientific, Inc.) following incubation with corresponding horseradish peroxidase (HRP)-conjugated antibodies. An automated chemiluminescence image analysis system (Tanon 6200; Tanon Science and Technology Co., Ltd., Shanghai, China) was used for protein visualization and to measure the optical density of protein bands. The primary antibodies used for experiments were as follows: anti-GAPDH (cat. no. KC-5G4; Kangchen Biotech), polyclonal rabbit anti-PRMT1 (cat. no. ab73246; Abcam, Cambridge, UK) and monoclonal rabbit anti-activated leukocyte cell adhesion molecule (ALCAM; cat. no. ab109215; Abcam). The corresponding anti-rabbit-HRP (cat. no. AP307P) and anti-mouse-HRP (cat. no. AP181P) antibodies (EMD Millipore) were used as secondary antibodies. All antibodies were diluted according to the manufacturer's instructions.

Cell proliferation assay. For the cell proliferation assay, cells were plated in 96-well plates at a density of 800 cells/well. Cell Counting Kit-8 assay (Dojindo Molecular Technologies, Inc., Kumamoto, Japan) was used to measure cell proliferation according to the manufacturer's protocol, and an Epoch microplate reader (BioTek Instruments, Inc., Winooski, VT, USA) was used to detect optical density values at $450 \mathrm{~nm}$ at the same time point of each day for 6 days.

In vivo tumor growth assay. $\mathrm{A} 375 / \mathrm{shNC}$ or A375/shPRMT1 cells $\left(1 \times 10^{6}\right.$ in $0.2 \mathrm{ml}$ sterilized phosphate-buffered saline) were implanted subcutaneously into the right flank of 4-week-old male BALB/c nude mice (five mice per group; weight, $\sim 20 \mathrm{~g}$ ). The mice were maintained in a specific pathogen-free environment at $20-22^{\circ} \mathrm{C}$ (12-h light/dark cycle) and provided with ad libitum access to food and autoclaved water. Tumor volume was calculated every 7 days using the following formula: $\mathrm{V}=$ (length) $\mathrm{x}$ (width) $\mathrm{x}$ (width)/2. The subcutaneous tumors were weighed following excision. The mice were housed in a specific pathogen-free environment and all experiments were performed in accordance with the official recommendations of the Chinese animal community.

Colony formation assay. For colony formation assays, cells from each transfection group in the logarithmic growth phase were incubated in 6-well plates at a density of 1,000 cells/well for 14 days and clones were stained with $1 \%$ crystal violet for counting.

Cell migration assay. Cell migration ability was assessed by Transwell assay (Corning Incorporated, Corning, NY, USA). Briefly, $1 \times 10^{5}$ cells from each group were added into the upper chamber of the Transwell containing $200 \mu 1$ serum-free RPMI-1640 medium with tumor supernatant of each group and $600 \mu \mathrm{l}$ DMEM containing 10\% FBS was added into lower chamber. Following incubation at $37^{\circ} \mathrm{C}$ and $5 \% \mathrm{CO}_{2}$ for $24 \mathrm{~h}$, cells that migrated to the underside of upper chamber were fixed with $1 \%$ methanol for $1 \mathrm{~h}$ at room temperature, then stained by $0.1 \%$ crystal violet, and counted in 5 random fields using a light microscope.

Reverse transcription-quantitative polymerase chain reaction (RT- $q P C R$ ) and qPCR array. TRIzol reagent (Invitrogen; Thermo Fisher Scientific, Inc.) was used for total RNA extraction from cell lines. cDNA was synthesized from $1 \mu \mathrm{g}$ of total RNA using a two-step method with a ReverTra Ace qPCR RT kit (Toyobo Co., Ltd., Osaka, Japan) according to the manufacturer's protocol. SYBR Green PCR Core reagent kit and ABI 7900 real-time PCR system (Applied Biosystems; Thermo Fisher Scientific, Inc.) were used for qPCR reactions. The primers used are as follows: PRMT1, forward 5'-CTT TGACTCCTACGCACACTT-3', and reverse 5'-GTGCCG GTTATGAAACATGGA-3'; GAPDH, forward 5'-AGCCAC 
A

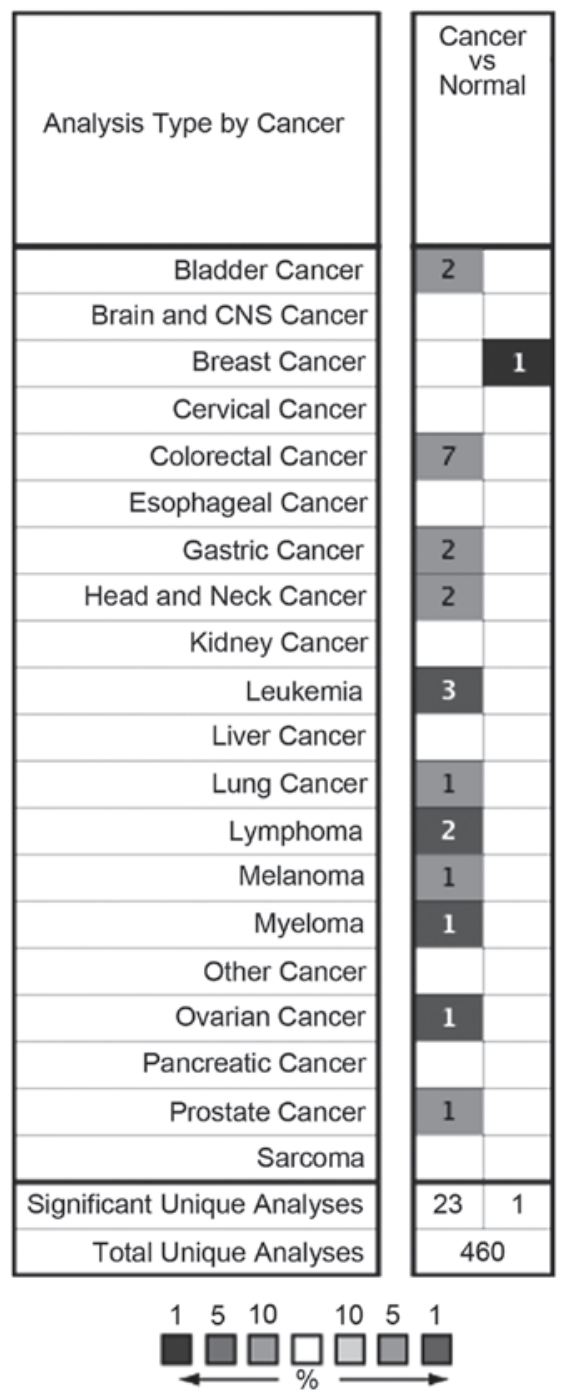

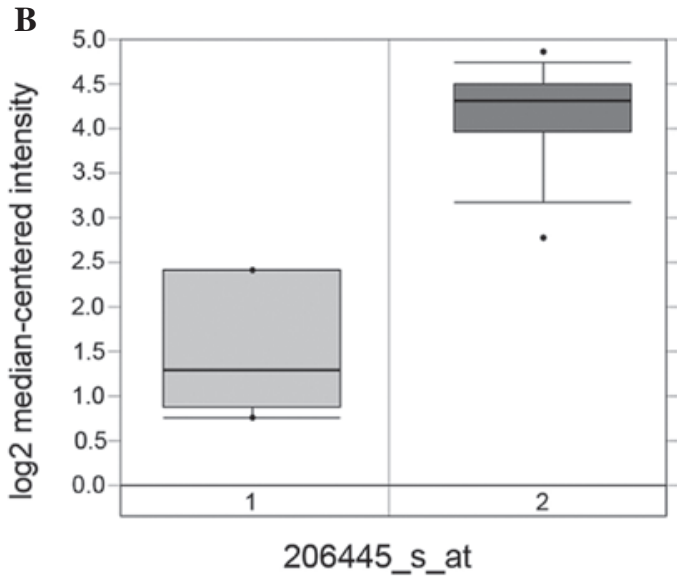
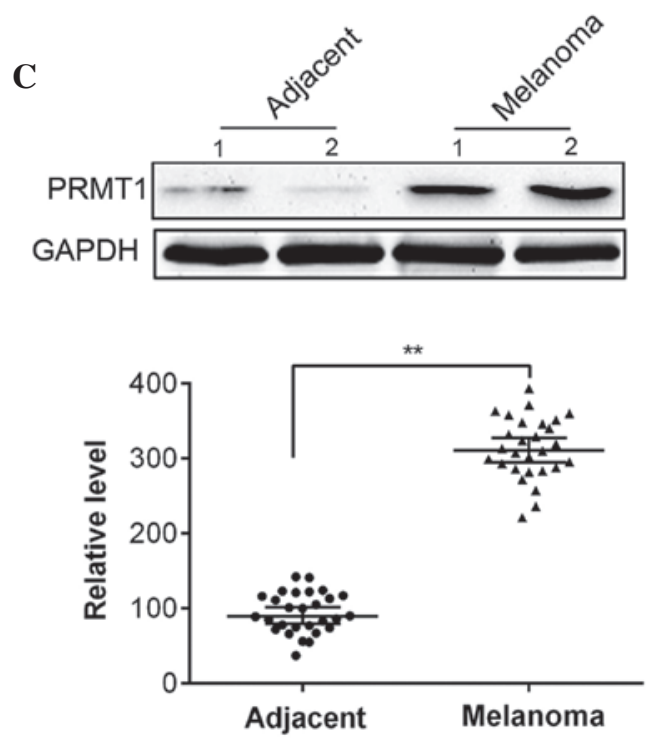

Figure 1. PRMT1 was overexpressed in human melanoma tissues by screening the Oncomine database (www.oncomine.org). (A) Overexpression of PRMT1 was found in 11 out of 20 cancer types, whereas downregulation of PRMT1 was only observed in breast cancer. (B) The mRNA level of PRMT1 was significantly increased in tumor tissues compared with non-tumor tissues in Talantov's dataset. (C) PRMT1 protein level in melanoma tissues and adjacent nontumorous tissues $(\mathrm{n}=28)$ was determined by western blot analysis. ${ }^{* *} \mathrm{P}<0.01$, comparison indicated by brackets. PRMT1, protein arginine methyltransferase 1 .

ATCGCTCAGACAC-3', and reverse 5'-GCCCAATACGAC CAAATCC-3'. Total RNA from the A375, A375/shNC and A375/shPRMT1 cells were used to perform a qPCR array by wcgene (Shanghai, China) according to the manufacturer's protocol. The relative mRNA levels were evaluated using the $2^{-\Delta \Delta \mathrm{Cq}}$ method (17).

Transient transfection. pcDNA3.1-EGFP-ALCAM or control plasmids (GeneChem, Inc., Daejeon, Korea) were transiently transfected into A375/shPRMT1 or Hs294T/shPRMT1 cells. Small interfering RNA (siRNA) targeting ALCAM (Shanghai GenePharma Co., Ltd., Shanghai, China) was employed for RNA interference (RNAi) experiments. The sequence of the ALCAM targeting siRNA was 5'-GUAAUU GUCCACUGAAUGGCUGGC-3' (sense). Briefly, cells were seeded in 6-well plates at a density of $3 \times 10^{5}$ cells/well and transfected with the corresponding plasmids/siRNA using Lipofectamine 2000 (Invitrogen; Thermo Fisher Scientific, Inc.) according to the manufacturer's protocol. Western blotting was performed to determine the transfection efficiency.
All functional studies were performed following transfection for $48 \mathrm{~h}$.

Statistical analysis. Student's t-test and one-way analysis of variance were used where appropriate. Data are presented as the mean \pm standard deviation from three independent experiments. SPSS software (version 15.0; SPSS, Inc., Chicago, IL, USA) was used to perform statistical analyses. $\mathrm{P}<0.05$ was considered to indicate a statistically significant difference.

\section{Results}

Identification of PRMT1 as a potential biomarker by screening the Oncomine database. Using the Oncomine database (www.oncomine.org), overexpression of PRMT1 was demonstrated in 11 out of 20 types of cancer types compared with normal tissue, whereas downregulation of PRMT1 was only demonstrated in breast cancer (Fig. 1A). In melanoma, PRMT1 was significantly overexpressed in Talantov's dataset (Fig. 1B). Furthermore, the protein level 

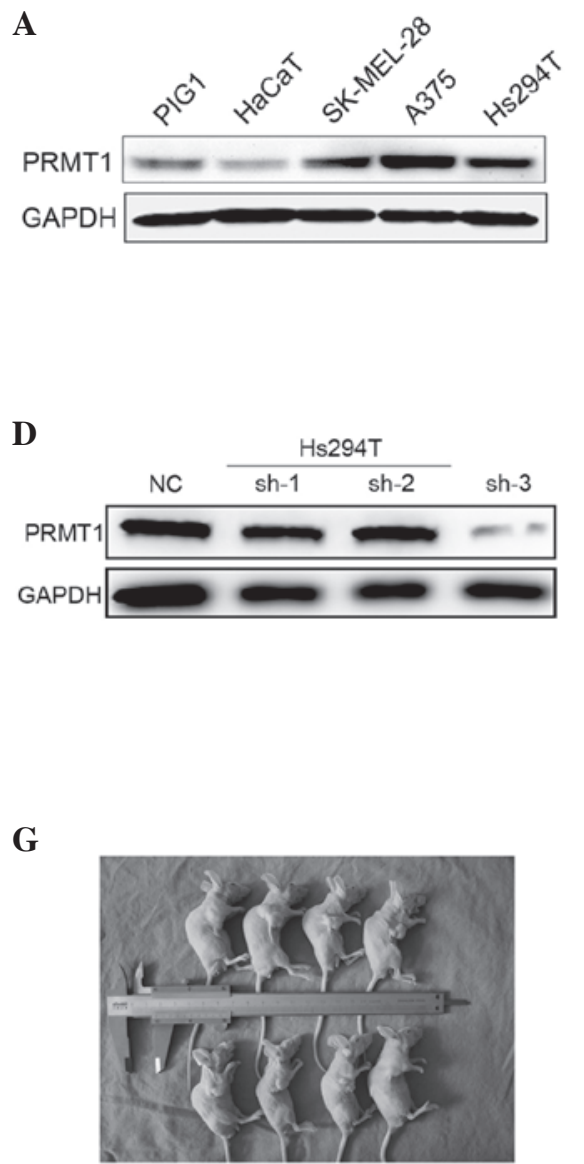

B

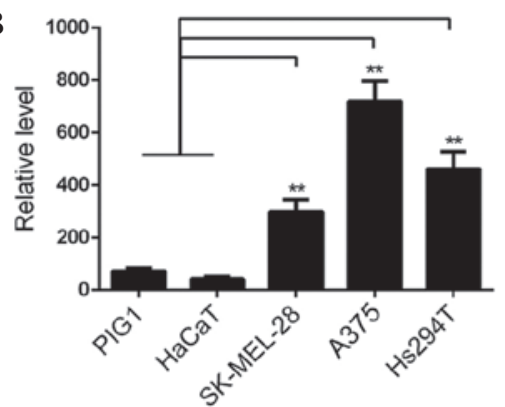

$\mathbf{E}$

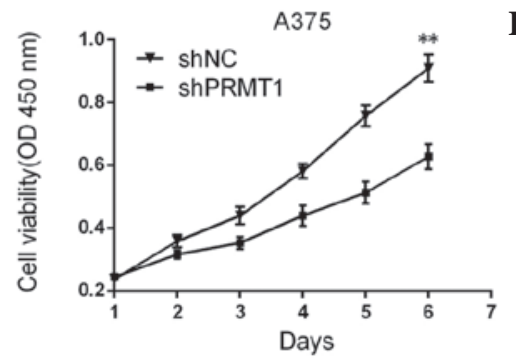

$\mathbf{H}$

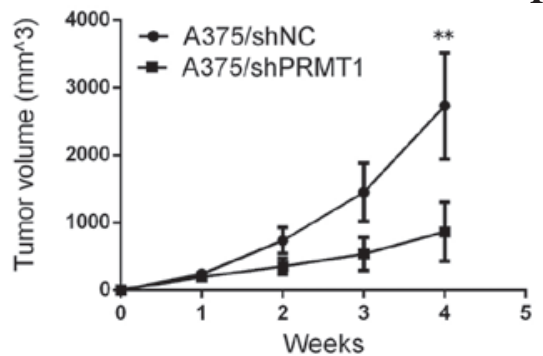

C

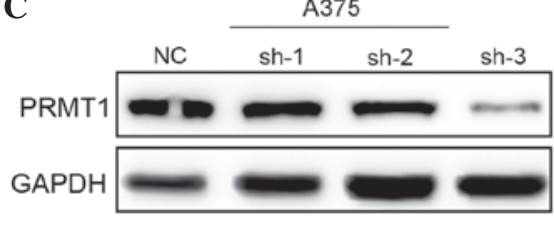

F
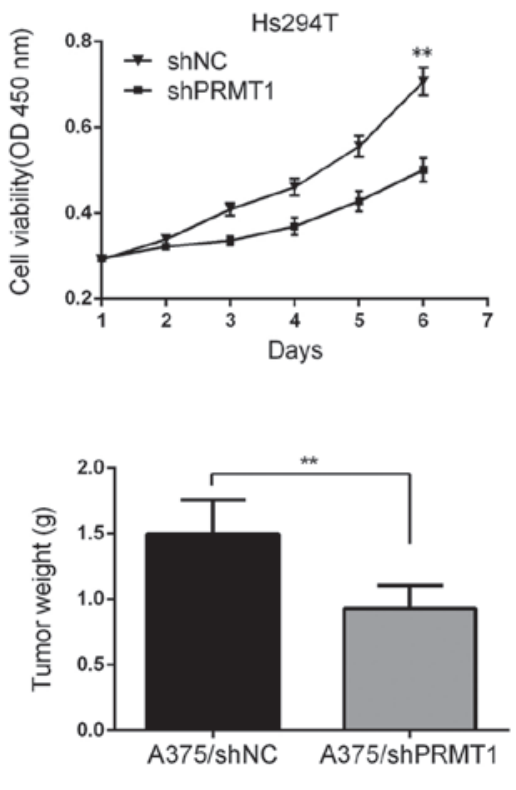

Figure 2. Silencing PRMT1 inhibited tumor growth in vitro and in vivo. (A and B) The protein level of PRMT1 was evaluated in a panel of melanoma cell lines compared with human immortalized keratinocytes or the immortalized human melanocyte cell line, PIG1. * P $<0.01$, comparison indicated by brackets. (C and D) The interference effect of A375 cells and Hs294T cells were confirmed by western blot analysis and sh-3 was identified as an effective interference shRNA for silencing PRMT1. (E and F) The results of cell counting kit-8 assay indicated that cell proliferation rate was significantly decreased after PRMT1 downregulation in A375 and Hs294T cells. ${ }^{* *} \mathrm{P}<0.01$. (G-I) Tumor growth and tumor size were significantly inhibited after PRMT1 knockdown in A375 cells. ${ }^{* *} \mathrm{P}<0.01$. PRMT1, protein arginine methyltransferase 1; NC, negative control; sh, short hairpin RNA.

of PRMT1 in 28 melanoma tissues and paired non-tumor tissues was determined. The present study demonstrated that the protein expression level of PRMT1 was significantly upregulated in melanoma compared with adjacent normal tissues $(\mathrm{P}<0.01$; Fig. 1C).

PRMT1 regulates cell proliferation in vitro and in vivo. The protein expression level of PRMT1 was determined by western blotting in several melanoma cell lines. It was demonstrated that the PRMT1 level was significantly higher in three melanoma cell lines (SK-MEL-28, A375 and Hs294T) compared with $\mathrm{HaCaTs}$ or immortalized human melanocyte cell line, PIG1 (P<0.01; Fig. 2A and B). Additionally, the effect of PRMT1 silencing on cell proliferation was analyzed. Of the three different shRNAs used, western blotting demonstrated that sh-3 yielded an efficient silencing effect on PRMT levels (Fig. 2C and D). The proliferative ability of A375 and Hs294T cells were determined at the same point on each day for 6 days. As indicated in Fig. 2E and F, silencing of PRMT1 significantly inhibited the proliferation of the cell lines $(\mathrm{P}<0.01)$. As PRMT1 was demonstrated to regulate in vitro cell proliferation, the effect of PRMT1 on tumor growth in vivo was investigated. A375 cells of each group were subcutaneously injected into nude mice. As demonstrated in Fig. 2G-I, knockdown of PRMT1 in A375 cells reduced tumor size and tumor growth compared with A375/shNC cells.

PRMT1 regulates colony formation, metastasis and multiple downstream cancer-associated genes. Plate colony formation assay was performed to determine the regulatory effect of PRMT1 on colony formation. As demonstrated in Fig. 3A, cells transfected with shNC exhibited significantly increased colony formation compared with the shPRMT1 group $(\mathrm{P}<0.01)$. Furthermore, the effect of PRMT1 on migration was investigated. A migration assay was performed using A375 and Hs294T cells transfected with shPRMT1 or shNC. As demonstrated in Fig. 3B, knockdown of PRMT1 significantly reduced cell migration through Transwell chambers $(\mathrm{P}<0.01)$. To understand the potential mechanisms that mediate the effect of PRMT1 on tumor growth and metastasis, a qPCR array including 24 genes was performed to investigate the changes that occur following PRMT1 silencing. Compared with shNC cells, A375/shPRMT1 cells exhibited significantly increased mRNA levels of cadherin 1 (CDH1), which is a well-known metastatic inhibitor. However, the mRNA levels of vascular endothelial growth factor (VEGF), matrix metalloproteinase 2 
A

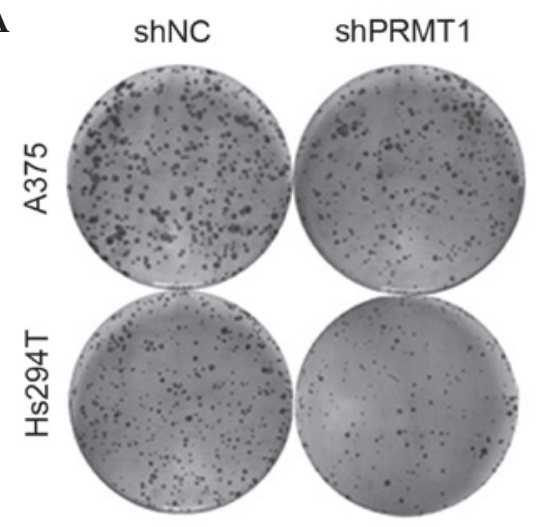

B

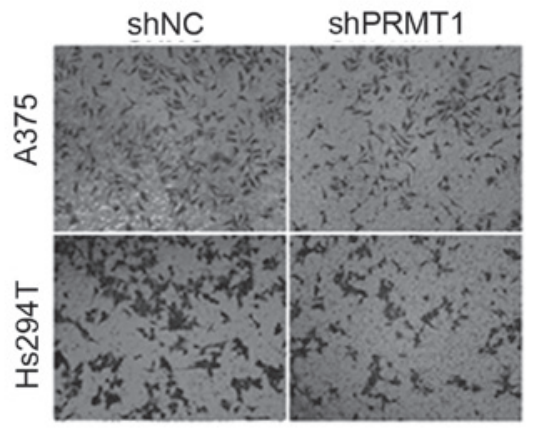

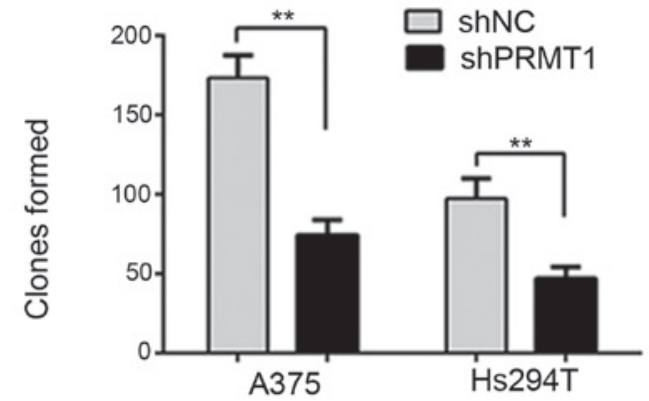

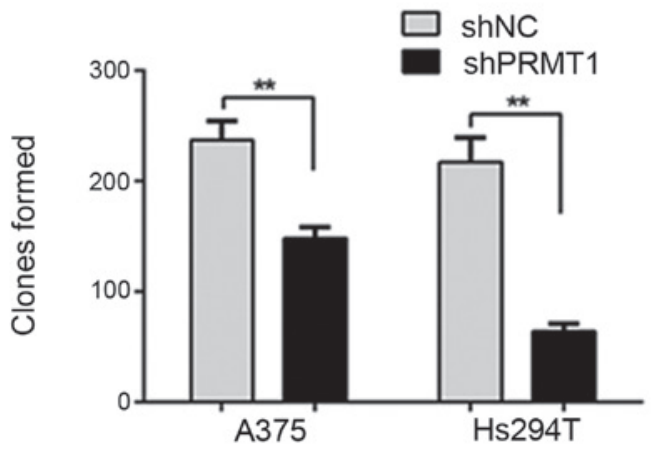

C

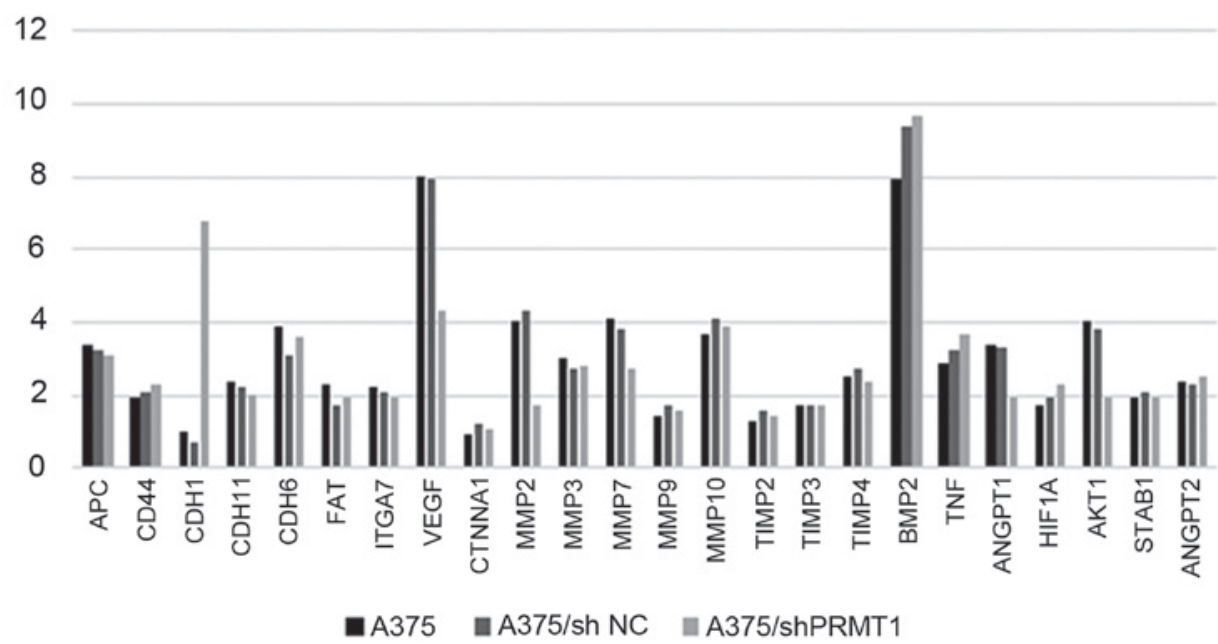

Figure 3. (A) Silencing PRMT1 inhibited colony formation ability of A375 cells and Hs294T cells. ${ }^{* *} \mathrm{P}<0.01$, comparison indicated by brackets. (B) The migration ability between A375/shNC and A375/shPRMT1, Hs294T/shNC and Hs294T/shPRMT1 was analyzed by Transwell analysis. The results indicated that silencing PRMT1 inhibited the metastatic potential of A375 cells and Hs294T cells. ${ }^{* *} \mathrm{P}<0.01$, comparison indicated by brackets. (C) mRNA level of CDH1 was upregulated and the level of VEGF, MMP2, AKT1 and ANGPT1 was downregulated by quantitative polymerase chain reaction array. sh, short hairpin RNA; $\mathrm{NC}$, negative control; PRMT1, protein arginine methyltransferase.

(MMP2), v-akt murine thymoma viral oncogene homolog 1 (AKT1) and angiopoietin 1 (ANGPT1) were downregulated in A375/shPRMT1 cells compared with A375/shNC cells (fold change, >1.5; Fig. 3C). VEGF and ANGPT1 are important angiogenic factors involved in tumor growth and metastasis of human melanoma. MMP2 is a member of the MMP protein family involved in angiogenesis, wound healing, tumor invasion and fibrosis. AKT1 is an important protein of the phosphatidylinositol-4,5-bisphosphate 3-kinase/AKT signaling pathway. Taken together, the changes in the mRNA levels of these genes provided a preliminary mechanism of PRMT1-induced tumor growth.
PRMT1 is a direct regulator of ALCAM and influence multiple aggressive behaviors. The current study demonstrated that PRMT1 regulates tumor progression and multiple downstream metastasis-associated genes; thus, proteins that interact with PRMT1 were further investigated using LC-MS and co-IP. The results of the present study indicated that ALCAM, a well-established oncogene in multiple human tumors, including human melanoma, interacts with PRMT1, as demonstrated by LC-MS and validated by co-IP (Fig. 4A and B). Additionally the current study investigated whether ALCAM is an upstream regulator of PRMT1 or a downstream co-effector. Silencing of PRMT1 expression in A375 and Hs294T cells 


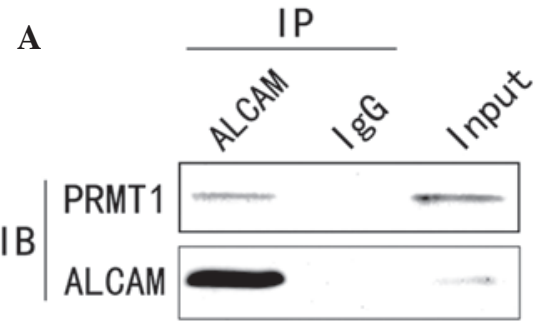

D

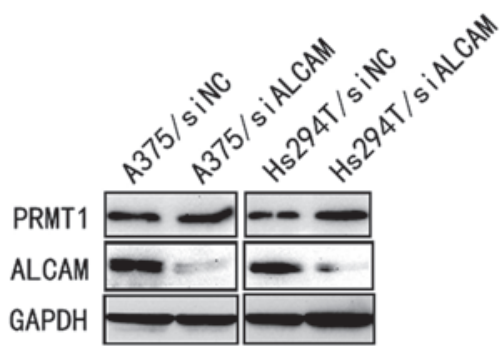

G

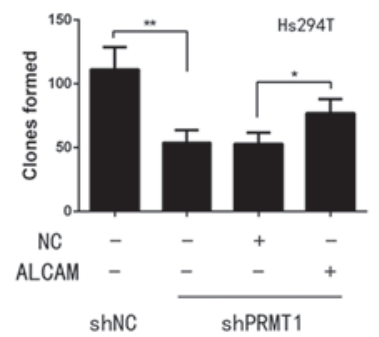

B

IB

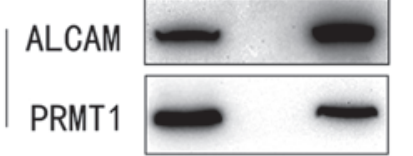

$\mathbf{E}$

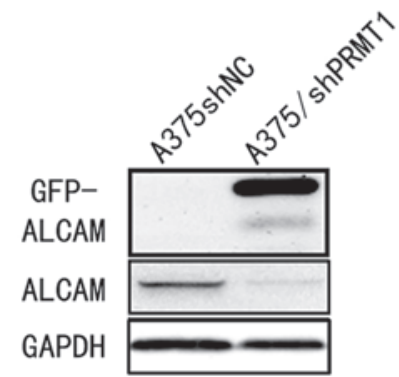

$\mathbf{H}$

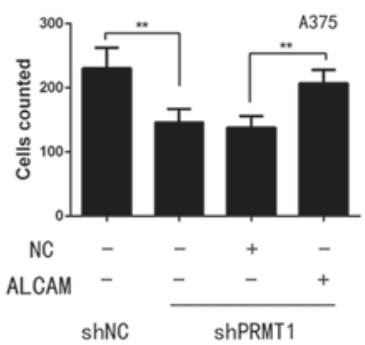

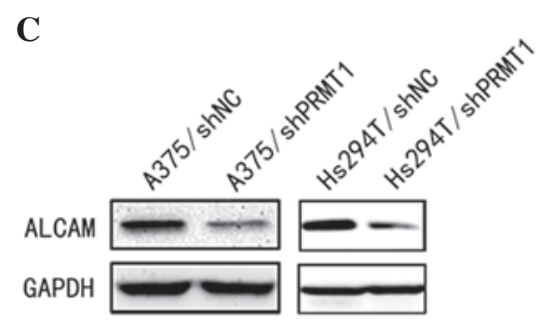

F

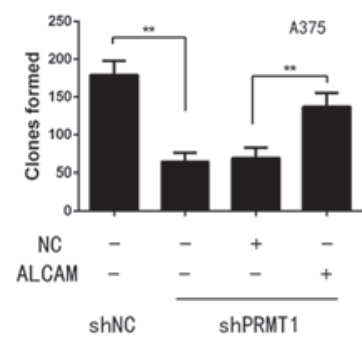

I

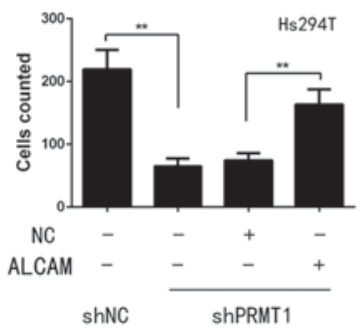

Figure 4. ALCAM was a direct target of PRMT1 and mediated multiple aggressive behaviors induced by PRMT1. (A and B) The interaction between PRMT1 and ALCAM was confirmed by co-IP. (C and D) Silencing PRMT1 in A375 and Hs294T cells could significantly downregulate ALCAM level while silencing ALCAM in A375 and Hs294T cells did not effect PRMT1 level. (E) pcDNA3.1-EGFP-ALCAM was transfected into A375/shPRMT1 cells and the transfection effect was confirmed by western blotting. (F and G) Colony formation ability of A375/shPRMT1 and Hs294T/shPRMT1 cells was significantly increased after ALCAM restoration. ${ }^{*} \mathrm{P}<0.05,{ }^{* *} \mathrm{P}<0.01$, comparison indicated by brackets. (H and I) Migration ability of A375/shPRMT1 and Hs294T/shPRMT1 cells was significantly reversed after ALCAM re-expression. ${ }^{* *} \mathrm{P}<0.01$, comparison indicated by brackets. IP, immunoprecipitation; ALCAM, activated leukocyte cell adhesion molecule; IgG, immunoglobulin G; IB, immunoblot; PRMT, protein arginine methyltransferase; sh, short hairpin RNA; NC, negative control; si, small interfering RNA.

downregulated the protein level of ALCAM compared with shNC (Fig. 4C). However, silencing of ALCAM using siRNA did not observably affect PRMT1 expression in A375 and Hs294T cells (Fig. 4D). These results indicated that ALCAM is a downstream target of PRMT1. Furthermore, silencing ALCAM inhibited cell proliferation (data not shown), which was in accordance with the phenotypes induced by silencing of PRMT1. To explore whether ALCAM was involved in PRMT1-induced tumor growth and metastasis, an ALCAM overexpression vector, pcDNA3.1-EGFP-ALCAM, was transfected into A375/shPRMT1 and Hs294T/shPRMT1 cells. ALCAM was fused with enhanced green fluorescent protein and expressed as a fusion protein. Western blot analysis demonstrated that transfection of A375/shPRMT1 with pcDNA3.1-EGFP-ALCAM resulted in overexpression of ALCAM (Fig. 4E). The functional analysis indicated that re-expression of ALCAM in shPRMT1 cells reversed the effects of PRMT1 silencing on the colony formation and migration ability compared with cells not transfected with the ALCAM expression plasmid (Fig. 4F-I). These results indicated that PRMT1 regulates tumor progression and metastasis via directly targeting ALCAM.

\section{Discussion}

The carcinogenesis of human melanoma involves complex genomic alterations and molecular processes (18). Typically, a malignant tumor undergoes the following stages during development: Precancerous stage; carcinoma in situ; and metastasis. For human melanoma, lymphatic vessel density in primary melanomas is correlated with the risk of metastasis in patients $(19,20)$. Furthermore, previous studies have preliminarily investigated the mechanisms that regulate the aggressive behavior of human melanoma, particularly the oncogenic function of several epigenetic regulators, including PRMTs (21-23). However, accurate molecular markers, and effective targets to clarify the clinical and biological features of melanoma, remain an urgent requirement.

Overexpression of PRMTs had been demonstrated in various types of human tumor (24). In the present study, screening of an online database (Oncomine) demonstrated that PRMT1 is overexpressed in human melanoma, which subsequently was confirmed by the experimental results. Thus, the biological function of PRMT1 in A374 and Hs294T cells was investigated. The current study demonstrated 
that silencing PRMT1 significantly inhibited tumor growth in vitro and in vivo, which is in accordance with the results of colony formation assays. Furthermore, PRMT1 silencing inhibited the metastatic potential of A375 and Hs294T cells, demonstrated by migration assay. To preliminarily investigate the altered phenotype following PRMT silencing, a qPCR array containing 26 genes was performed to detect potential downstream metastasis-associated genes. The mRNA level of CDH1, a metastatic inhibitor, was downregulated, whereas the mRNA level of VEGF, MMP2, AKT1 and ANGPT1 were upregulated following silencing of PRMT1 in A375 cells. These results provided direct evidence that PRMT1 regulates multiple downstream tumorigenesis markers.

Additionally, the mechanisms mediate PRMT1-regulated tumor growth and metastasis were investigated. Protein was extracted from A375/shNC and A375/shPRMT1 cells and immunoprecipitated with anti-PRMT1 antibody for LC-MS analysis. LC-MS demonstrated that ALCAM may be a potential downstream co-effector of PRMT1. The negative association between the ALCAM level and the survival status of patients with colorectal cancer and esophageal squamous cell carcinoma has been previously reported $(25,26)$. In human melanoma, the ALCAM level was previously demonstrated to be correlated with tumor progression, and regulates melanoma cell clustering and migration (27-29). The association between PRMT1 and ALCAM was demonstrated by co-IP. Furthermore, the current study demonstrated that silencing of PRMT1 in A375 cells significantly downregulates the ALCAM level. Furthermore, A375/shPRMT1 and Hs294T/shPRMT1 cells were transfected with pcDNA3.1-EGFP-ALCAM to determine whether ALCAM restoration affects the phenotypes induced by PRMT1 silencing. Notably, cells in the shPRMT1 group exhibited significantly increased colony formation and metastatic ability following restoration of ALCAM expression. Taken together, the results of the present study demonstrate that ALCAM is a direct target of PRMT1 and involved in PRMT1-induced tumorigenesis.

In conclusion, the results of the current study indicate that PRMT1 is overexpressed in human melanoma, and that PRMT1 may regulate tumor growth and metastasis via targeting ALCAM. The PRMT1-ALCAM axis may be a novel anti-cancer target for the treatment of human melanoma.

\section{Acknowledgements}

This study was supported by Medical Science and Technology Project in Henan province (grant. no. 201403165).

\section{References}

1. Siegel R, Naishadham D and Jemal A: Cancer statistics, 2013. CA Cancer J Clin 63: 11-30, 2013.

2. Itakura E, Huang RR, Wen DR, PaulE, Wünsch PH and Cochran AJ: IL-10 expression by primary tumor cells correlates with melanoma progression from radial to vertical growth phase and development of metastatic competence. Mod Pathol 24: 801-809, 2011.

3. Gandini S, Sera F, Cattaruzza MS, Pasquini P, Picconi O, Boyle P and Melchi CF: Meta-analysis of risk factors for cutaneous melanoma: II. Sun exposure. Eur J Cancer 41: 45-60, 2005.

4. Shi H, Hugo W, Kong X, Hong A, Koya RC, Moriceau G, Chodon T, Guo R, Johnson DB, Dahlman KB, et al: Acquired resistance and clonal evolution in melanoma during BRAF inhibitor therapy. Cancer Discov 4: 80-93, 2014.
5. Puig-Butille JA, Escámez MJ, Garcia-Garcia F, Tell-Marti G, Fabra Â, Martínez-Santamaría L, Badenas C, Aguilera P, Pevida M, Dopazo J, et al: Capturing the biological impact of CDKN2A and MC1R genes as an early predisposing event in melanoma and non melanoma skin cancer. Oncotarget 5: 1439-1451, 2014

6. Garbe C, Peris K, Hauschild A, Saiag P, Middleton M, Spatz A, Grob JJ, Malvehy J, Newton-Bishop J, Stratigos A, et al: Diagnosis and treatment of melanoma: European consensus-based interdisciplinary guideline. Eur J Cancer 46: 270-283, 2010.

7. Loquai C, Schmidtmann I, Beutel M, Sunderkötter C, Grabbe S, Schiller M and Nashan D: Quality of life in melanoma patients during adjuvant treatment with pegylated interferon- $\alpha 2 b$ : Patients' and doctors' views. Eur J Dermatol 21: 976-984, 2011.

8. Dillman RO, DePriest C, Ellis R and de Leon C: 5-year survival for patients with metastatic melanoma who had no evidence of disease at time of treatment with patient specific tumor stem cell vaccines. Cancer Research 74: 197, 2014.

9. Di Lorenzo A and Bedford MT: Histone arginine methylation. FEBS Lett 585: 2024-2031, 2011.

10. Wysocka J, Allis CD and Coonrod S: Histone arginine methylation and its dynamic regulation. Front Biosci 11: 344-355, 2006.

11. Yang Y and Bedford MT: Protein arginine methyltransferases and cancer. Nat Rev Cancer 13: 37-50, 2013.

12. Gu Z, Gao S, Zhang F, Wang Z, Ma W, Davis RE and Wang Z: Protein arginine methyltransferase 5 is essential for growth of lung cancer cells. Biochem J 446: 235-241, 2012.

13. Yoshimoto T, Boehm M, Olive M, Crook MF, San H, Langenickel T and Nabel EG: The arginine methyltransferase PRMT2 binds RB and regulates E2F function. Exp Cell Res 312: 2040-2053, 2006.

14. Qi C, Chang J, Zhu Y, Yeldandi AV, Rao SM and Zhu YJ: Identification of protein arginine methyltransferase 2 as a coactivator for estrogen receptor alpha. J Biol Chem 277: 28624-28630, 2002.

15. Nicholas C, Yang J, Peters SB, Bill MA, Baiocchi RA, Yan F, Sif S, Tae S, Gaudio E, Wu X, et al: PRMT5 is upregulated in malignant and metastatic melanoma and regulates expression of MITF and p27 (Kip1.). PLoS One 8: e74710, 2013.

16. Nicholas C, Yan F, Peters SB, Bill MA, Li P, Li C, Fuchs JR, Baiocchi R and Lesinski GB: The expression of PRMT5 methyltransferase mediates cell survival and metastatic phenotype in malignant melanoma. Cancer Research 71: 933, 2011.

17. Livak KJ and Schmittgen TD: Analysis of relative gene expression data using real-time quantitative PCR and the 2(-Delta Delta C(T)) Method. Methods 25: 402-408, 2001.

18. Hodis E, Watson IR, Kryukov GV, Arold ST, Imielinski M, Theurillat JP, Nickerson E, Auclair D, Li L, Place C, et al: A landscape of driver mutations in melanoma. Cell 150: 251-263, 2012.

19. Shayan R, Karnezis T, Murali R, Wilmott JS, Ashton MW, Taylor GI, Thompson JF, Hersey P, Achen MG, Scolyer RA and Stacker SA: Lymphatic vessel density in primary melanomas predicts sentinel lymph node status and risk of metastasis. Histopathology 61: 702-710, 2012.

20. Shields CL, Kaliki S, Furuta M, Fulco E, Alarcon C and Shields JA: American Joint Committee on Cancer classification of posterior uveal melanoma (tumor size category) predicts prognosis in 7731 patients. Ophthalmology 120: 2066-2071, 2013.

21. Chapman PB, Hauschild A, Robert C, Haanen JB, Ascierto P, Larkin J, Dummer R, Garbe C, Testori A, Maio M, et al: Improved survival with vemurafenib in melanoma with BRAF V600E mutation. N Engl J Med 364: 2507-2516, 2011.

22. Huang FW, Hodis E, Xu MJ, Kryukov GV, Chin L and Garraway LA: Highly recurrent TERT promoter mutations in human melanoma. Science 339: 957-959, 2013.

23. Krauthammer M, Kong Y, Ha BH, Evans P, Bacchiocchi A, McCusker JP, Cheng E, Davis MJ, Goh G, Choi M, et al: Exome sequencing identifies recurrent somatic RAC1 mutations in melanoma. Nat Genet 44: 1006-1014, 2012.

24. Bao X, Zhao S, Liu T, Liu Y, Liu Y and Yang X: Overexpression of PRMT5 promotes tumor cell growth and is associated with poor disease prognosis in epithelial ovarian cancer. J Histochem Cytochem 61: 206-217, 2013.

25. Weichert W, Knösel T, Bellach J, Dietel M and Kristiansen G: ALCAM/CD166 is overexpressed in colorectal carcinoma and correlates with shortened patient survival. J Clin Pathol 57: 1160-1164, 2004. 
26. Verma A, Shukla NK, Deo SV, Gupta SD and Ralhan R: MEMD/ALCAM: A potential marker for tumor invasion and nodal metastasis in esophageal squamous cell carcinoma. Oncology 68: 462-470, 2005.

27. Degen WG, Van Kempen LC, Gijzen EG, van Groningen JJ, van Kooyk Y, Bloemers HP and Swart GW: MEMD, a new cell adhesion molecule in metastasizing human melanoma cell lines, is identical to ALCAM (activated leukocyte cell adhesion molecule). Am J Pathol 152: 805-813, 1998.
28. Swart GW, Lunter PC, Kilsdonk JW and Kempen LC: Activated leukocyte cell adhesion molecule (ALCAM/CD166): Signaling at the divide of melanoma cell clustering and cell migration? Cancer Metastasis Rev 24: 223-236, 2005.

29. Lunter PC, Van Kilsdonk JW, Van Beek H, Cornelissen IM, Bergers M, Willems PH, van Muijen GN and Swart GW: Activated leukocyte cell adhesion molecule (ALCAM/CD166/MEMD), a novel actor in invasive growth, controls matrix metalloproteinase activity. Cancer Res 65: 8801-8808, 2005. 